\title{
Ectima gangrenoso, manifestación cutánea infrecuente de una infección por diversos agentes
}

\author{
(Ecthyma gangrenosum, a rare cutaneous manifestation of infection by \\ various agents)
}

Nanco-Meléndez C. ${ }^{*}$, López-Mora E. ${ }^{2}$, Cruz-Choappa R.

${ }^{1}$ Residente Dermatología, Universidad De Los Andes, Santiago, Chile.

${ }^{2}$ Residente Infectología, Universidad De Valparaíso, Valparaíso, Chile

${ }^{3}$ Infectología, Universidad De Valparaíso, Valparaíso, Chile.

*Autor para correspondencia: cnanco@miuandes.cl

RECIBIDO: 10 de enero 2021

APROBADO: 10 de febrero 2021

DOI: $10.22370 /$ bolmicol.2020.35.2.2715

Palabras claves: Ectima gangrenoso, Pseudomonas aeruginosa, Sepsis.

Keywords: Ecthyma gangrenosum, Pseudomonas aeruginosa, Sepsis.

\section{RESUMEN}

El ectima gangrenoso es un trastorno infeccioso infrecuente clásicamente relacionado a bacteriemia, descrito principalmente en poblaciones inmunodeprimidas. El agente más comúnmente relacionado es Pseudomonas aeruginosa, sin embargo, se han descrito otras etiologías bacterianas, hongos filamentosos y levaduras. Su patogénesis está dada por la invasión de la pared de los vasos sanguíneos, causando trombosis arterial y venosa, desencadenando necrosis de epidermis y tejidos subdérmicos. Clínicamente, se manifiesta como máculas, vesículas o pústulas hemorrágicas que evolucionan a úlceras de superficie necrótica rodeadas por un halo eritematoso característico, habitualmente en un contexto clínico de sepsis. El diagnóstico de este cuadro es clínico, sin embargo, el estudio microbiológico es clave en la identificación del agente etiológico y un posterior tratamiento dirigido. En su manejo es esencial una alta sospecha clínica y el inicio de tratamiento antibiótico en forma precoz. La duración del tratamiento es variable y en algunos casos debe asociarse a debridación quirúrgica. El pronóstico es variable dependiendo de múltiples factores: estado inmunológico, agente etiológico, presencia de sepsis y el tiempo de inicio de tratamiento.

ABSTRACT
Ecthyma gangrenosum is an infrequent
infectious disorder classically related to
bacteremia, described mainly in
immunosuppressed populations. The most
related agent is Pseudomonas aeruginosa,
however other bacterial etiologies, filamentous
fungi and yeasts have been described. Its
pathogenesis is given by the invasion of the
blood vessel wall, causing arterial and venous
thrombosis, triggering necrosis of epidermis and
subdermal tissues. Clinically, it manifests as


Ectima gangrenoso, manifestación cutánea infrecuente de una infección por diversos agentes - Nanco C.

hemorrhagic macules, vesicles or pustules that evolve into ulcers with a necrotic surface surrounded by a characteristic erythematous halo, usually in a clinical setting of sepsis. The diagnosis of this condition is clinical, however the microbiological study is key in the identification of the etiological agent and a subsequent directed treatment. In its management, a high clinical suspicion and an early start of antibiotic treatment are essential. The duration of treatment is variable and in some cases it must be associated with surgical debridement. The prognosis is variable depending on multiple factors: immunological status, etiological agent, presence of sepsis and time of initiation of treatment.

\section{INTRODUCCIÓN}

El ectima gangrenoso corresponde a un trastorno infeccioso descrito por L. Barker el año 1897 (1). Hasta 1970 fue considerado una manifestación patognomónica de bacteriemia secundaria a Pseudomona aeruginosa, particularmente en pacientes con inmunosupresión secundaria a neoplasia hematológica (2).

El ectima gangrenoso corresponde a una entidad infrecuente, sin datos específicos a nivel mundial, afecta cualquier rango etario, desde neonatos a adultos mayores, sin diferencias por raza o sexo (2).

Hoy en día se han descrito múltiples agentes causales de esta enfermedad, incluyendo no solo bacterias, sino que también a hongos. El año 2014 Vaiman et al. (3) realizaron una revisión sobre 167 casos reportados en la literatura, encontrado en el 76,5\% a $P$. aeruginosa como el agente causal, seguido en un $17,35 \%$ por otras bacterias, $\mathrm{y}$, finalmente, $9 \%$ especies fúngicas. $\mathrm{Si}$ bien existen casos asociados con virus herpes simple (VHS) y virus de inmunodeficiencia humana (VIH), la etiología viral como causante de ectima gangrenoso es aún controversial.

Otras bacterias que se han descrito asociadas a ectima gangrenoso son Aeromonas hydrophila, Pseudomona maltophilia y Escherichia coli.
Con menor frecuencia se han detectado otras especies de Pseudomonas y Mycobacterium tuberculosis. Las bacterias gram positivas, como Staphylococcus o Streptococcus, son causa infrecuente de esta enfermedad. Actualmente, solo 8 casos secundarios a Staphylococcus se han reportados en la literatura, siendo solo 3 sensibles a la meticilina (SAMS) (Imagen 1, 2 y 3) (4) (5) (6).

Por otro lado, y con menor frecuencia, los agentes micóticos asociados a ectima gangrenoso son hongos levaduriformes como Candida spp y hongos filamentosos como Fusarium spp (3) (7).

\section{Fisiopatología y patogenicidad}

Su patogénesis está dada por la invasión de los microorganismos en la pared de vasos sanguíneos, causando trombosis arterial y venosa, desencadenando infarto y necrosis de epidermis y tejidos subdérmicos, hecho que ocurre particularmente en los casos secundarios a bacteriemia (8) (9).

Característicamente, se ha descrito en pacientes con inmunidad alterada, no obstante, cada vez son más los casos en pacientes inmunocompetentes (10). Dentro de las causas de inmunosupresión más frecuentemente asociadas se encuentran las neoplasias hematológicas, seguido por malnutrición, quemaduras severas, trasplantados o usuarios de medicamentos inmunosupresores. El uso de antibióticos previos puede favorecer la infección por Pseudomonas spp (9).

Otras patologías concomitantes a esta enfermedad que se han publicado en la literatura son la presencia de diabetes, VIH, enfermedades del tejido conectivo y otras enfermedades infecciosas preexistentes (3). En niños puede ocurrir asociado a inmunodeficiencias, particularmente relacionadas con neutropenia (9).

Clínicamente, en la gran mayoría de los casos el paciente se encuentra séptico, con gran compromiso del estado general, no obstante, esta 
Ectima gangrenoso, manifestación cutánea infrecuente de una infección por diversos agentes - Ñanco C.

entidad se ha descrito en pacientes sanos sin bacteriemia o fungemia concomitante (11).

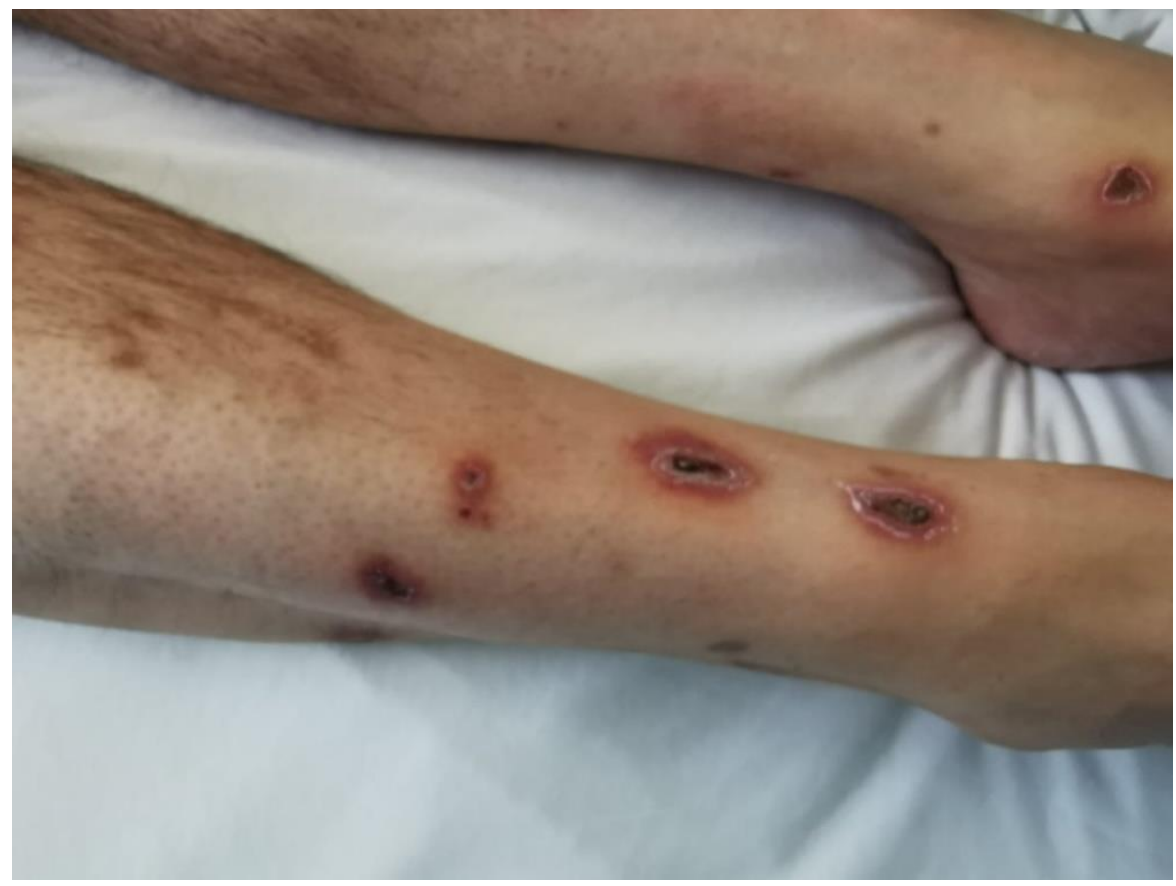

Imagen 1. Placas ovaladas con centro necrótico y halo eritematoso en extremidades inferiores en un paciente diabético con hemocultivos positivos para SAMS.

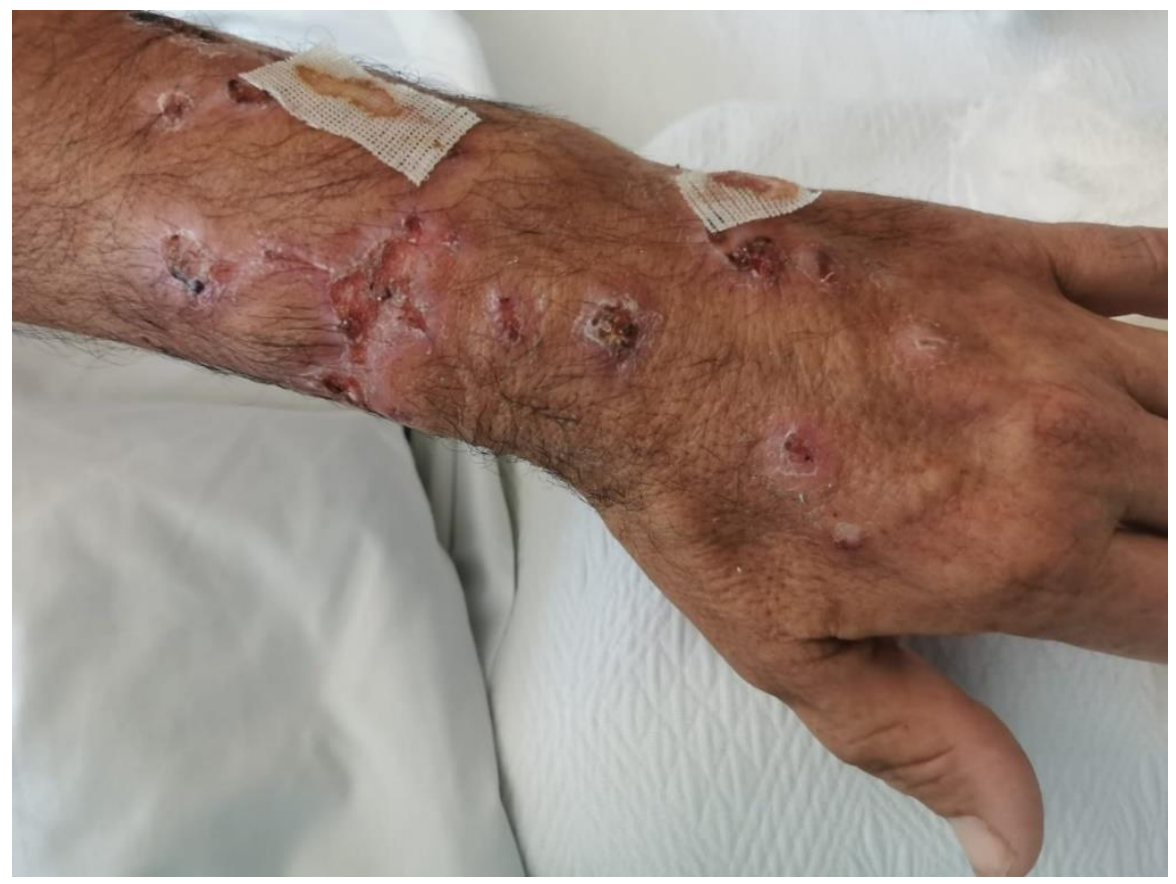

Imagen 2. Placas con centro necrótico y halo eritematoso en extremidad superior en un paciente diabético con hemocultivos positivos para SAMS. 
Ectima gangrenoso, manifestación cutánea infrecuente de una infección por diversos agentes - Nanco C.

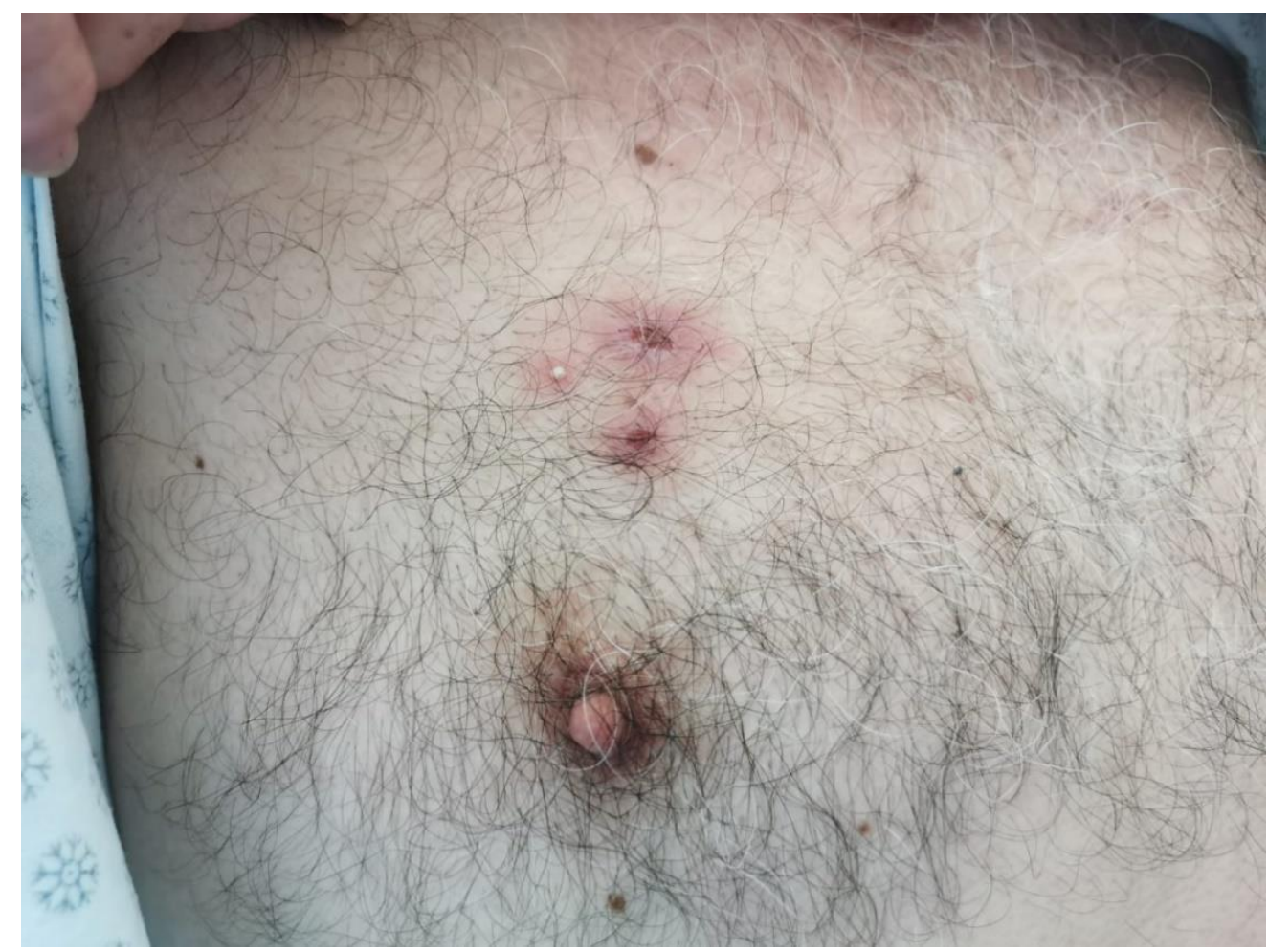

Imagen 3. Placas y pústulas con halo eritematoso en tronco en un paciente diabético con hemocultivos positivos para SAMS.

\section{Manifestaciones clínicas}

Desde el punto de vista cutáneo se aprecian máculas, vesículas o pústulas hemorrágicas que evolucionan a úlceras de superficie necrótica rodeadas por un halo eritematoso característico. Esta transición ocurre en un periodo variable, incluso entre 12 a 24 horas (9). Pueden ocurrir en cualquier lugar del cuerpo, siendo más frecuentes en región glútea y extremidades inferiores (3). En neonatos puede afectar la cara (12).

La presentación múltiple se asocia con diseminación hematógena del agente causal, mientras que lesiones aisladas son causadas por una inoculación directa en la piel afectada. Las lesiones causadas por S. aureus no difieren de la presentación clínica de ectima gangrenoso secundario a otros agentes (4) (5).

$\mathrm{Si}$ bien el diagnóstico es clínico, es de suma utilidad la toma de hemocultivos y biopsia cutánea, enviando esta última a estudio histológico y cultivo de bacterias, hongos y micobacterias (3). Cabe destacar que los hemocultivos pueden resultar negativos, lo cual es frecuente en los casos causados por $S$. aureus (4).

Histológicamente, se puede encontrar necrosis dérmica difusa, hallazgo similar a pioderma gangrenoso (9). En ocasiones es visible vasculitis en la capas externas e intermedias de vasos sanguíneos, pero sin afectar la capa íntima. Se ha descrito además epidermis necrótica, vénulas congestionadas, extravasación de glóbulos rojos, edema de la dermis papilar y separación de la unión dermo epidérmica (7).

El diagnóstico diferencial es amplio, debiéndose considerar otras causas de úlceras cutáneas como vasculitis, pioderma gangrenoso, loxoscelismo, fasceitis necrotizante, calcifilaxis, coagulación intravascular diseminada, vasculopatía livedoide y necrosis inducida por Warfarina (7). 
Ectima gangrenoso, manifestación cutánea infrecuente de una infección por diversos agentes - Nanco C.

\section{Tratamiento}

El pilar de tratamiento de esta enfermedad es estabilizar al paciente y el uso de terapia antibiótica empírica precoz, la cual debe ser ajustada posteriormente, según resultado de cultivos. Según la sospecha diagnóstica y los factores de riesgo del paciente, el tratamiento empírico es variable, siendo los más utilizados la ceftazidima, ampicilina, amoxicilina con ácido clavulánico, anfotericina B o voriconazol $(3,9)$. En los casos secundarios a SAMS es recomendable utilizar cloxacilina o cefalosporinas de primera generación. Por otro lado, en los casos por SAMR se debe ocupar vancomicina, cotrimoxazol o linezolid (4). La duración de tratamiento es variable, depende de la respuesta clínica y del agente aislado. En casos severos es necesario asociar al tratamiento antibiótico el abordaje quirúrgico, con el fin de debridar las escaras remanentes.

El pronóstico es variable según la inmunidad del paciente, el agente responsable, la presencia de sepsis y el tiempo en inicio de tratamiento. Se estima que la mortalidad es mayor en pacientes bacteriémicos y en usuarios que recibieron tratamiento antibiótico previo al desarrollo de la infección (9) (13).

\section{CONCLUSIÓN}

El ectima gangrenoso es un trastorno infeccioso grave que ocurre principalmente en inmunosuprimidos, no obstante, se ha visto en pacientes con otras comorbilidades, como la diabetes. Si bien es causado principalmente por Pseudomonas aeruginosa, otros agentes etiológicos han sido involucrados, dentro de los cuales se encuentra el $S$. aureus. Es fundamental la toma precoz de hemocultivos y cultivos bacterianos y/o micológicos de las lesiones, con el fin de determinar el agente etiológico responsable, seguido por el inicio de la terapia empírica precoz.

\section{REFERENCIAS}

1. Barker L. The clinical symptoms, bacteriologic findings and postmortem appearances in cases of infection of human beings with the Bacillus pyocyaneus. JAMA. 1897; 29:213-6.

2. Vaiman M, Lasarovitch T, Heller L, Lotan G. Ecthyma gangrenosum versus ecthyma-like lesions: should we separate these conditions? Acta Dermatovenerol Alp Pannonica Adriat. 2015;24(4):69-72.

3. Vaiman M, Lazarovitch T, Heller L, Lotan G. Ecthyma gangrenosum and ecthymalike lesions: review article. Eur J Clin Microbiol Infect Dis Off Publ Eur Soc Clin Microbiol. 2015;34(4):633-9.

4. Ivanaviciene J, Chirch L, Grant-Kels JM, Kerr PE, Finch J. Ecthyma gangrenosum secondary to methicillin-sensitive Staphylococcus aureus. Int $\mathrm{J}$ Womens Dermatol.2016;2(3):89-92.

5. Buffière-Morgado $\mathrm{A}$, Demongeot $\mathrm{C}$, Battistella M, Taieb F, Viguier M, Rybojad M, et al. [Ecthyma gangrenosum associated with infection involving a methicillin-sensitive, Panton-Valentine-negative strain of Staphylococcus aureus]. Ann Dermatol Venereol. 2015;142(4):262-5.

6. Jaque A, Moll-Manzur C, Dossi MT, Berroeta-Mauriziano D, Araos-Baeriswyl E, Monsalve X. Ectima gangrenoso secundario a Staphylococcus aureus. Rev Chil Infectol. 2016;33(3):336-9.

7. Jiang Y, Al-Hatmi AMS, Xiang Y, Cao Y, van den Ende AHGG, Curfs-Breuker I, et al. The Concept of Ecthyma Gangrenosum Illustrated by a Fusarium oxysporum Infection in an Immunocompetent Individual. Mycopathologia. 2016;181(9):759-63. 
8. Ceylan N, Demir N, Kocaman S, Peker E, Tuncer O. An Extraordinary Cause of the Sucking Difficulty: Ecthyma Gangrenosum. Case Rep Med [Internet]. 2016 [citado 24 de enero de 2021];2016. Disponible en: https://www.ncbi.nlm.nih.gov/pmc/articles/PM C4837260/

9. Wuyts L, Wojciechowski M, Maes P, Matthieu L, Lambert J, Aerts O. Juvenile ecthyma gangrenosum caused by Pseudomonas aeruginosa revealing an underlying neutropenia: case report and review of the literature. J Eur Acad Dermatol Venereol JEADV. 2019;33(4):781-5.

10. Fabrizi G, Pagliarello C. Multiple ecthyma gangrenosum with a favorable course in a nonimmunocompromised child with moyamoya disease. Eur J Dermatol EJD. 2007;17(3):253-4.

11. Gençer S, Özer S, Ege Gül A, Doğan M, $\mathrm{Ak}$ Ö. Ecthyma gangrenosum without bacteremia in a previously healthy man: a case report. J Med Case Reports. 2008; 2:14.

12. Pathak A, Singh P, Yadav Y, Dhaneria M. Ecthyma gangrenosum in a neonate: not always pseudomonas. BMJ Case Rep. 2013;2013.

13. Lee YJ, Jung IO, Oh DY. A Rare Case of Ecthyma Gangrenosum Caused by Proteus vulgaris and Candida albicans in a Patient with Castleman Disease. Arch Plast Surg. 2015;42(6):805-7 